\title{
Photoacoustic tomography with novel optical contrast agents based on gold nanocages or nanoparticles containing near-infrared dyes
}

Xinmai Yang, Sara Skrabalak, Erich Stein, Bin Wu, Xunbin Wei, et al.

Xinmai Yang, Sara Skrabalak, Erich Stein, Bin Wu, Xunbin Wei, Younan Xia, Lihong V. Wang, "Photoacoustic tomography with novel optical contrast agents based on gold nanocages or nanoparticles containing near-infrared dyes," Proc. SPIE 6856, Photons Plus Ultrasound: Imaging and Sensing 2008: The Ninth Conference on Biomedical Thermoacoustics, Optoacoustics, and Acousto-optics, 68560I (28 February 2008); doi: 10.1117/12.762401 


\title{
Photoacoustic tomography with novel optical contrast agents based on gold nanocages or nanoparticles containing near-infrared dyes
}

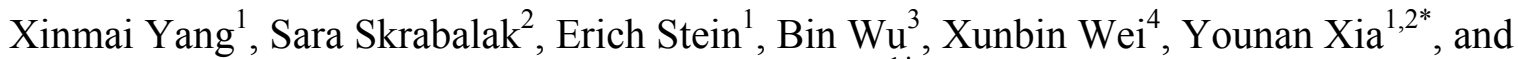 \\ Lihong V. Wang ${ }^{1 \dagger}$ \\ ${ }^{1}$ Optical Imaging Laboratory, Department of Biomedical Engineering, Washington University in St. \\ Louis, St. Louis, Missouri 63130 \\ ${ }^{2}$ Department of Chemistry, University of Washington - Seattle, WA 98195 \\ ${ }^{3}$ Phosphorex, Inc., Fall River, MA 02723 \\ ${ }^{4}$ Institutes of Biomedical Sciences, Fudan University, Shanghai, 200032 China
}

\begin{abstract}
Poly(ethylene glycol)-coated Au nanocages have been evaluated as a potential near-infrared (NIR) contrast agent for photoacoustic tomography (PAT). Previously, Au nanoshells were found to be an effective NIR contrast agent for PAT; however, Au nanocages, with their more compact sizes ( $<50 \mathrm{~nm}$ compared to $>100 \mathrm{~nm}$ for Au nanoshells) and larger optical absorption cross-sections, should be better suited for in vivo applications. In this study, we tested $\mathrm{Au}$ nanocages as a contrast agent for PAT. The result suggests that Au nanocages are promising contrast agents for our applications. We also present PAT results when novel, dye-containing nanoparticles are used as contrast agents.
\end{abstract}

Keywords: photoacoustic tomography, image reconstruction, nanocages, dye-containing nanoparticles.

\section{INTRODUCTION}

Photoacoustic tomography (PAT) is a novel, hybrid, and non-ionizing imaging modality that combines the merits of both purely optical imaging and ultrasonic imaging methods. It is also highly sensitive to the optical absorption of biological tissue. PAT provides greater spatial resolution than purely optical imaging in deep regions while simultaneously overcoming the disadvantages of ultrasonic imaging regarding both biochemical contrast and speckle artifact; it can provide high spatial resolution images with optical contrast in a region up to $5.2 \mathrm{~cm}$ deep. ${ }^{1}$

PAT has been successfully applied to the visualization of different structures in biological tissues and has been especially useful in imaging the cerebral cortex of small animals. ${ }^{2-6}$ PAT is also suitable for monitoring the circulation of exogeneous optical contrast agents. ${ }^{7,8}$ Such contrast agents have been shown to improve the sensitivity and specificity of optical imaging techniques such as optical coherent tomography (OCT), fluorescence imaging, and other microscopies. ${ }^{9}$ Still, purely optical imaging techniques cannot provide high spatial resolution in regions beyond $1 \mathrm{~mm}$ deep because of the strong scattering from biological tissues.

Long-circulating, optically tunable nanoparticles have recently been applied to PAT for use as contrast agents, allowing for their uptake, delivery, and excretion from a body to be monitored. The results from such monitoring could aid in the diagnosis of cancer. For example, these small (typically 60 to $400 \mathrm{~nm}$ diameter) particles tend to extravate and accumulate in tumor regions via a passive mechanism referred to as the "enhanced permeability and retention effect," which has been attributed to dysfunctional anatomical conditions such as localized leaky circulatory and lymphatic

\footnotetext{
*Email: xia@biomed.wustl.edu

†Email: lhwang@biomed.wustl.edu
}

Photons Plus Ultrasound: Imaging and Sensing 2008: The Ninth Conference on Biomedical Thermoacoustics, Optoacoustics, and Acousto-optics, edited by Alexander A. Oraevsky, Lihong V. Wang, Proc. of SPIE Vol. 6856, 68560I, (2008) · 1605-7422/08/\$18 - doi: 10.1117/12.762401 
systems. This feature is especially helpful in brain tumor detection since healthy vessels in the brain dissuade the extravasation of such particles because of the blood-brain barrier. As a result, nanoparticles can accumulate in the vicinity of tumor vasculature and greatly enhance NIR optical contrast. In a previous study, Au nanoshells were tested as a NIR optical contrast agent in PAT. In this study, we have sought to evaluate a new class of optically tunable nanoparticles, Au nanocages, as a potential NIR contrast agent for PAT.

This work aims to tailor the optical properties of the Au nanocages for use with PAT. To this end, we have prepared Au nanocages with an average edge length of $\sim 50 \mathrm{~nm}$. Discrete dipole approximation (DDA) calculations indicate that these small, hollow Au nanostructures have a particularly large absorption cross-section, nearly 5 orders of magnitude greater than that of conventional organic dyes. ${ }^{10}$ This feature is attractive to absorption-based imaging techniques such as PAT. Specifically, we show the feasibility of Au nanocages as an intravascular contrast agent for PAT of small animals in vivo. The vasculature of a rat's cerebral cortex was imaged, with enhanced optical contrast being observed with the use of Au nanocages.

We also tested NIR dyes-containing nanoparticles as PAT contrast agents. Cabocyanine dyes are the most widely used NIR optical probes for imaging tumors in small animals and humans. They have exceptionally high molar absorptivity (typically $10^{5} \mathrm{M}^{-1} \mathrm{~cm}^{-1}$ ). Among cabocyanine dyes, indocyanine green (ICG) is particularly useful because it has been proved by the U.S. Food and Drug Administration for clinic applications. It has been used in various imaging applications such as cardiac output monitoring, hepatic function study, peripheral circulation assessment and tumor detection. However, ICG has several disadvantages. First, when administered intravenously, ICG has a plasmatic halflife of 2-4 minutes and shows extensive protein binding. The short circulation time and protein binding prevent it from effective use as a fluorescent probe for optical imaging. Second, ICG is degraded in aqueous solution and the degradation is accelerated by light and heat. The degradation makes it impossible to prepare a stable ICG bio-conjugate in the aqueous media. Consequently, several ICG derivatives have been developed. These derivatives are reactive and are more stable in aqueous media. However, the toxicity and biocompatibility of these ICG derivatives have not been fully investigated.

Phosphorex, Inc. has long been developing and commercializing near-IR nanoparticles. The nanoparticles Phosphorex developed have sizes ranging from $50 \mathrm{~nm}$ to $500 \mathrm{~nm}$. At Phosphorex, ICG and other near-IR dyes have been encapsulated in various polymer matrixes to form nano-sized particles or spheres. Here we present the PAT results using these nanopaticles as contrast agents.

\section{METHODS AND MATERIALS}

Au nanocages with an edge length of $\sim 50 \mathrm{~nm}$ were prepared in a two-step process. First, Ag nanocubes were prepared by a modified polyol process. ${ }^{11} 6.0 \mathrm{~mL}$ of ethylene glycol (EG, J.T. Baker 9300-01 Lot C42B27) was added to a glass vial and heated at $152^{\circ} \mathrm{C}$ for 1 hour while being stirred with a Teflon-coated magnetic stir bar at 260 rpm's. $90 \mu \mathrm{L}$ of a $3 \mathrm{mM}$ sodium sulfide (J. T. Baker 3910)-EG solution was then injected into the vial. After 8.5 minutes, $1.5 \mathrm{~mL}$ of a PVP $(20 \mathrm{mg} / \mathrm{mL}$, J. T. Baker, cat. no. 3910, MW = 29,000)-EG solution was injected into the vial. Immediately thereafter, $0.50 \mathrm{~mL}$ of a $\mathrm{AgNO}_{3}(48 \mathrm{mg} / \mathrm{mL}$, Sigma-Aldrich 209139)-EG solution was injected into the vial. A series of color changes were observed over the next 12 minutes, with the reaction being stopped by cooling the vial to room temperature after the reaction media appeared opaque, green-ochre when viewed head-on and ruddy-red when viewed from the top. Plating was observed on the vial walls. The quenched reaction media was then diluted twice its volume with acetone and the Ag nanocubes were collected by centrifugation. The Ag nanocubes were resuspended in water and washed an additional 3 times then stored in $4 \mathrm{~mL}$ of deionized water for future use.

The Ag nanocubes were converted into Au nanocages via the galvanic replacement reaction. $400 \mu \mathrm{L}$ of the $\mathrm{Ag}$ nanocubes were dispersed in $10 \mathrm{~mL}$ water containing $1 \mathrm{mg} / \mathrm{mL}$ PVP in a $50 \mathrm{~mL}$ flask under magnetic stirring. The suspension was heated to a gentle reflux ( $\sim 10$ minutes). Then $5.4 \mathrm{~mL}$ of a $0.5 \mathrm{mM} \mathrm{HAuC}_{14}$ aqueous solution was added to the flask via syringe pump at a rate of $0.75 \mathrm{~mL} / \mathrm{min}$. Upon completion of $\mathrm{HAuCl} 4$ solution addition, the suspension appeared dark blue. The suspension remained heated for an additional 10 minutes. Once cooled to room temperature, the sample was saturated with $\mathrm{NaCl}$ (to remove $\mathrm{AgCl}$ ) then spun down. The supernatant was discarded and the sample resuspended in water. The sample was washed 6 additional times to remove PVP and $\mathrm{NaCl}$. The Au nanocage optical 


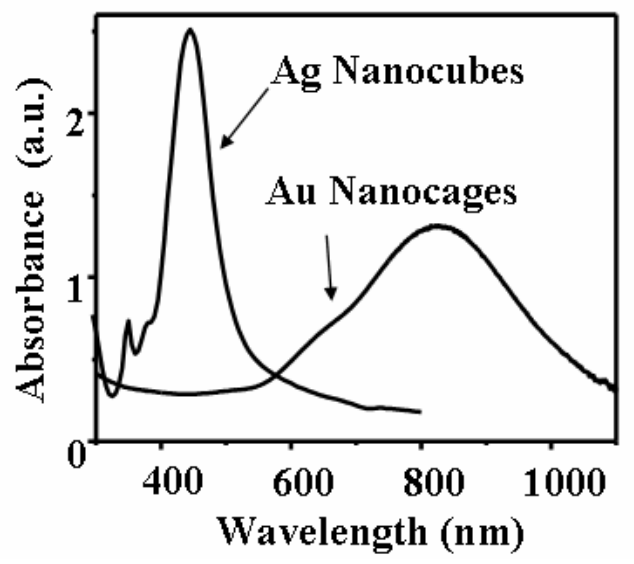

Figure 1. Absorption spectra of Ag nanocubes and Au nanocages.

properties were assessed using a UV-visible spectrophotometer. The resulting Au nanocages had a peak optical extinction (absorption and scattering) at $\sim 820 \mathrm{~nm}$ (Figure 1), a wavelength that overlaps with the optically transparent window of biological tissues and the laser employed in the PAT experiments.

The concentration of Ag nanocubes was determined by correlating atomic emission spectroscopy with SEM, with $\sim 10$ $\%$ of the anticipated Au nanocages being lost during washing. The Au nanocages were then functionalized with poly(ethylene glycol) (PEG), which has been shown to suppress immunogenic responses and thus improve blood circulation times. $^{12,13}$ Au nanocage surfaces were functionalized with PEG by adding $1 \mathrm{~mL}$ of a $1 \mathrm{mM} \mathrm{mPEG}-\mathrm{SH}$ (5000 MW PEG-SH, Nektar)-water solution to a $2 \mathrm{nM}$ cage suspension. The suspension was gently agitated then allowed to sit undisturbed overnight. Residual mPEG-SH was removed by centrifugation.

Dye-containing nanoparticles were obtained from Phosphorex, Inc. At Phosphorex, ICG has been encapsulated in various polymer matrixes to form ICG nanoparticles. These nanoparticles are received from Phosphorex, Inc. and tested in our PAT imaging system. The properties of these nanoparticles are listed in the above table (Table 1). 
Table 1 Phosphorex Dye-Containing Nanoparticles

\begin{tabular}{|l|l|l|}
\hline Sample ID & $\begin{array}{l}\text { Effective Dye Conc. } \\
(\mathrm{mg} / \mathrm{ml})\end{array}$ & $\begin{array}{l}\text { Recommended } \\
\text { Injection Volume } \\
(\mathrm{ml} / 100 \mathrm{~g})\end{array}$ \\
\hline NIRex $^{\mathrm{TM}}-4002$ & 300 & 0.25 \\
\hline NIRex $^{\mathrm{TM}}-4004$ & 125 & 0.6 \\
\hline
\end{tabular}

The setup for noninvasive photoacoustic tomography of rat brains is shown in Figure 2. A Q-switched Nd:YAG laser (LS-2137/2, LOTISTII)-pumped tunable Ti:sapphire laser (LT-2211A, LOTIS TII) was employed to provide laser pulses with a FWHM $<15 \mathrm{~ns}$, a pulse repetition rate of $10 \mathrm{~Hz}$, and a wavelength of $804 \mathrm{~nm}$. The incident energy density of the laser beam was controlled to be less than $10 \mathrm{~mJ} / \mathrm{cm}^{2}$ on the surface of the rat head, which is well below the ANSI limit $\left(32 \mathrm{~mJ} / \mathrm{cm}^{2}\right.$ at $\left.804 \mathrm{~nm}\right)$. An unfocused ultrasonic transducer (V310, Panametrics) with a central frequency of $10 \mathrm{MHz}$ and a $-6 \mathrm{~dB}$ bandwidth of about $70 \%$ was used to detect the ultrasound signals. The rat was fixed with a homemade mount so that its head protruded into the water tank through a hole in the tank's bottom, with a piece of polyethylene film sealing the gap between the water and the rat's head. Ultrasonic coupling gel was applied on the surface of the rat head. The photoacoustic signals detected by the ultrasonic transducer were received by an amplifier and then sent to an oscilloscope. A computer collected the digitized signals to reconstruct the distribution of optical

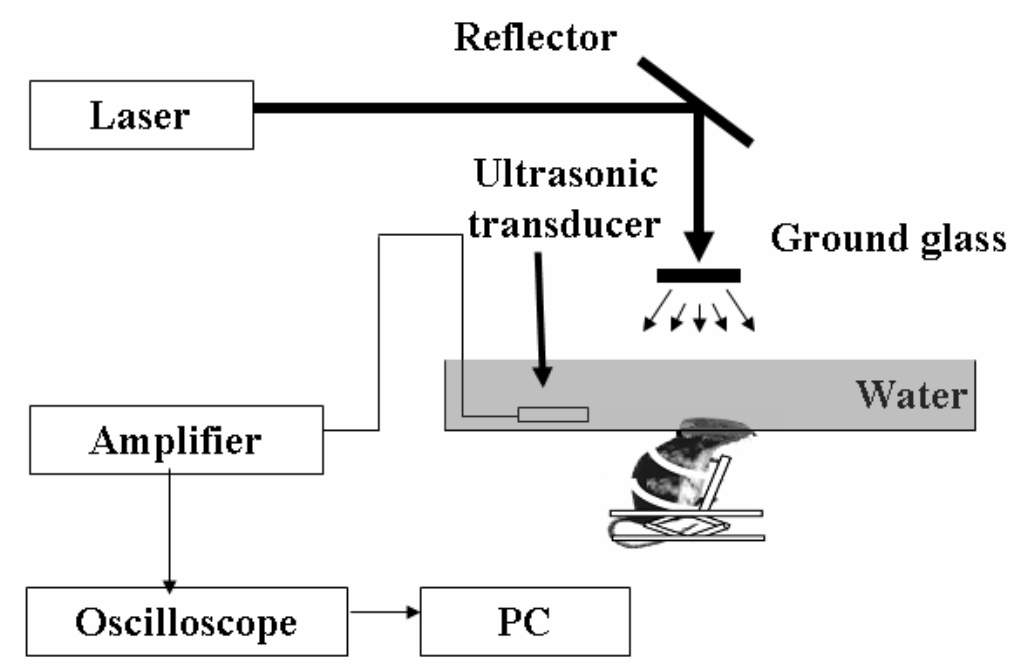

Figure 2 Experimental setup 
absorption in the imaging plane through a modified backprojection algorithm. ${ }^{14}$

Sprague Dawley rats (100-150 g, Harlan Sprague Dawley, Inc., Indianapolis, Indiana) were employed for the imaging experiments. Before imaging, the hair on each rat's head was removed with hair removal lotion. A dose of $85 \mathrm{mg} / \mathrm{kg}$ Ketamine plus $15 \mathrm{mg} / \mathrm{kg}$ Xylasine was administered intramuscularly to anesthetize the rats. The subsequent anesthesia was achieved by the inhalation of a mixture of $\mathrm{O}_{2}$ and isoflourane. The heart rate and blood oxygenation $\left(\mathrm{SpO}_{2}\right)$ level were monitored by a pulse oximeter. The heart rate of the animals was $\sim 300 \mathrm{bpm}$ and the $\mathrm{SpO}_{2}$ level was $>98 \%$ during the data acquisition.

The nanocages were injected into the circulatory system of the rats through the tail veins or saphenous veins. Three successive injections of nanocages were administrated, each with a dose of $\sim 0.8 \times 10^{9}$ nanocages $/ \mathrm{g}$ body weight. PAT scanning started immediately following each administration. After three administrations, PAT scanning was continuously performed for up to 6 hours. After data acquisition, the rat was sacrificed with an injection of concentrated pentobarbital. Open skull surgery was performed to photograph the cerebral cortex. With another animal, a single injection with a dose of $\sim 0.8 \times 10^{9}$ nanocages/g body weight was also performed, and PAT scanning immediately followed the injection and continued for about 3 hours. For dye-containing nanoparticles, only one injection was used for all test.

A)

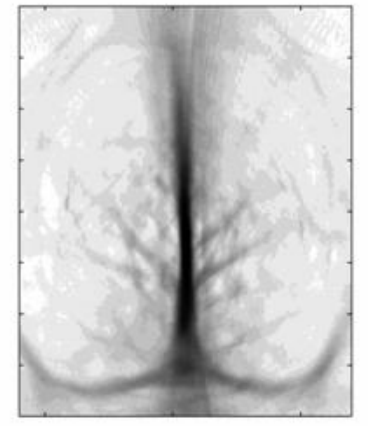

B)

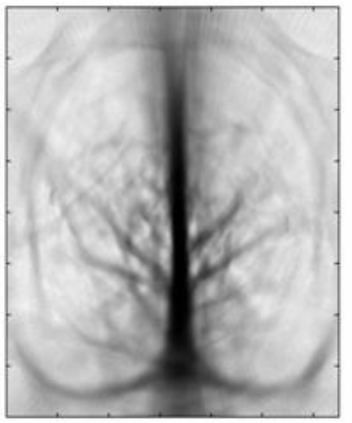

c)

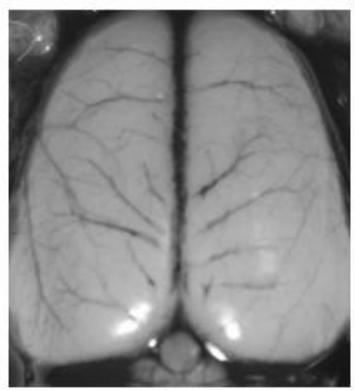

Figure 3 Non-invasive PAT imaging of a rat's cerebral cortex (A) before the injection of nanocages; (B) about 2 hours after the final injection of nanocages, which is the peak enhancement point. (D) An open-skull photograph of the rat's cerebral cortex. Three successive injections were administrated in these results.

\section{RESULTS}




\subsection{Results for Au Nanocacges}

PAT images of the cerebral cortex of a rat of the three successive injections are presented in Figures 3A and 3B with the same color bars. Figure 3A shows the cerebral cortex imaging before the injection of nanocages while Figure 3B is after the injection of nanocages. Compared to Figure 3A in which only intrinsic optical contrast provides the image, Figure 3B, acquired after the administration of Au nanocages, shows the brain vasculature with much greater clarity. With $\mathrm{Au}$ nanocages, the optical absorption of the blood was increased and the contrast between the vessels and the background brain tissues was enhanced. All the PAT images of the rat brain match well with the open-skull anatomical photograph shown in Figure 3C.

A)

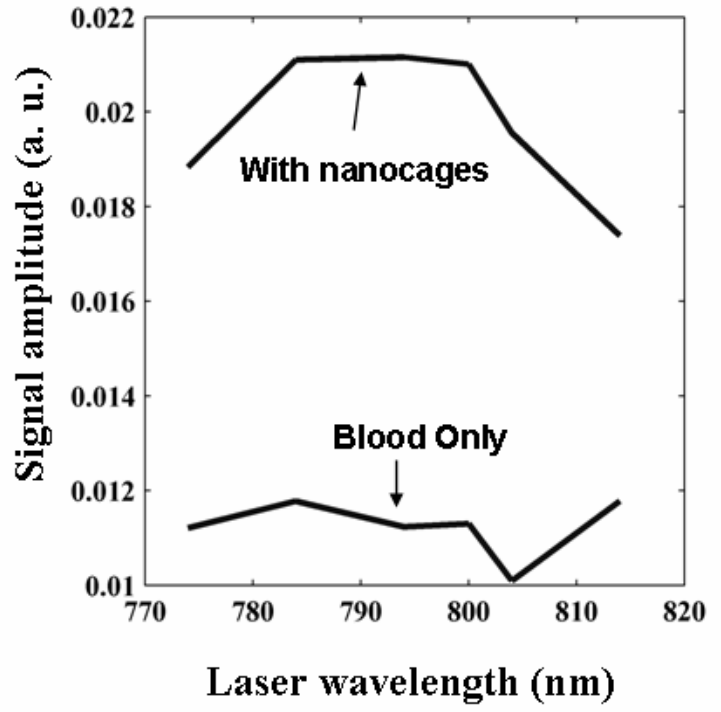

B)

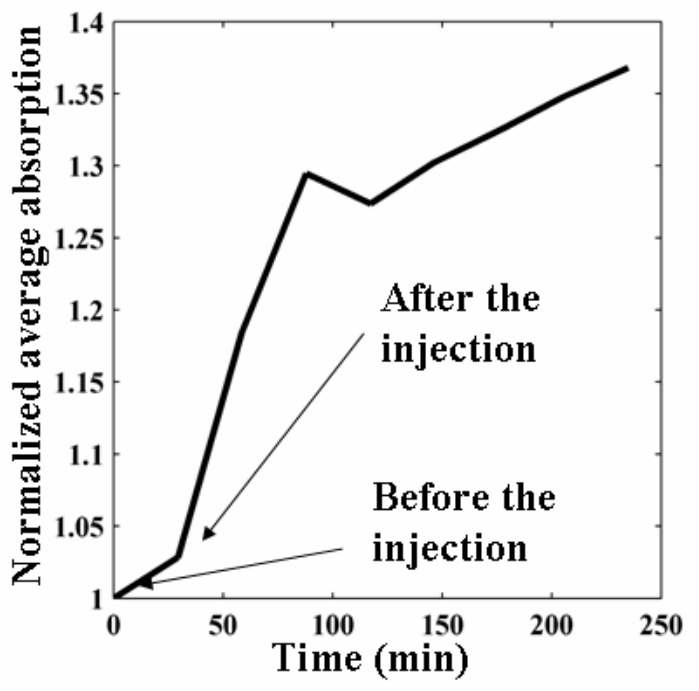

Figure 4 A) Ex vivo measurements of optical absorption spectral of rat blood with and without $\mathrm{Au}$ nanocages. B) The integrated absorption calculated from the experimental images for the singleinjection experiment. The presented values are normalized by the integrated absorption of the image obtained before the injection. The error bars are the standard errors for each integration

The absorption spectrum of the nanocages mixed with rat blood was also measured ex vivo by detecting photoacoustic signals. First, nanocages were mixed with rat blood at a ratio equivalent to $\sim 0.8 \times 10^{9}$ nanocages/g body weight, and injected into a small Tygon tube $(0.94 \mathrm{~mm}$ OD, $51 \mathrm{~mm}$ ID). The tube was then illuminated by the laser while the generated photoacoustic signals were detected with a ultrasonic transducer. The laser wavelength was tuned from 764 $\mathrm{nm}$ to $824 \mathrm{~nm}$, and the photoacoustic signals were collected. As a reference, the absorption spectrum for pure rat blood was also measured. This result is shown in Figure 4A. Figure 4A shows that with blood only, the amplitudes of measured photoacoustic signals are considerably lower. After adding the Au nanocages into the blood, the amplitudes increase by $85-106 \%$ over this wavelength range. The increases in optical absorption in vivo were also calculated for all PAT images. Figure 4B shows the enhancement result for a single injection. In Figure 4B, the enhancements have a peak value of $35 \%$, which occurs about 2 hours after the injection. 
A)

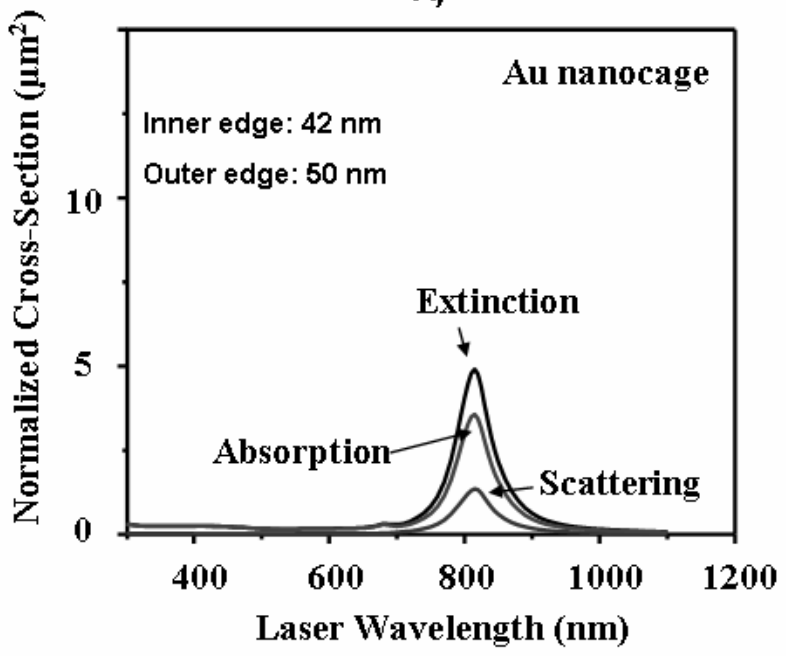

C)

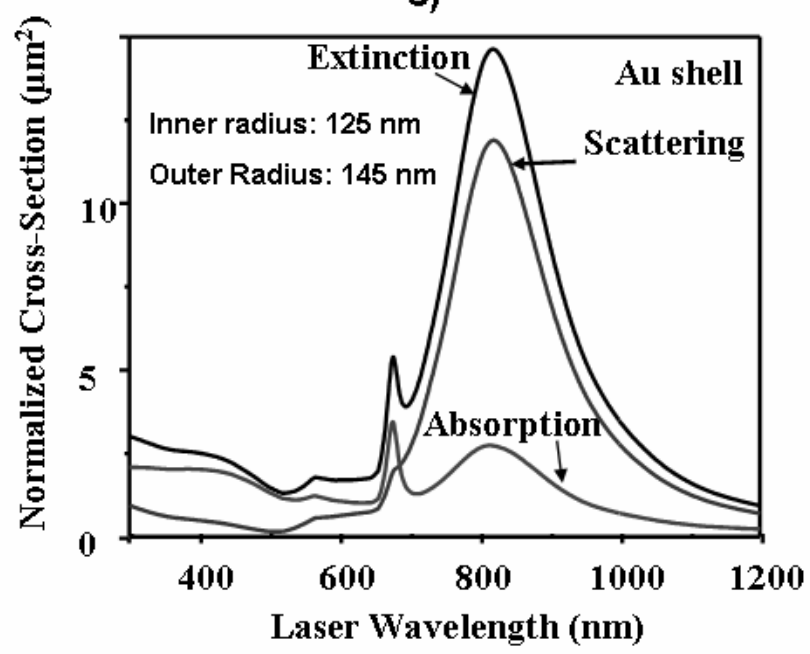

Figure 5 Extinction spectra calculated using Mie theory and the discrete dipole approximation (DDA) method for (A) a Au nanoshell composed of a $\mathrm{SiO}_{2}$ core (diameter $=125 \mathrm{~nm}$ ) and 10-nm thick Au shell and (B) a Au nanocage with an edge length of $50 \mathrm{~nm}$ and wall thickness of $4 \mathrm{~nm}$, respectively. Both were simulated to be in a water environment.

To further compare Au nanoshells and Au nanocages, we simulated the extinction spectra for both nanoparticles in water using the DDA approach. The parameters used in the simulation were $\mathrm{D}_{\text {out }}=145 \mathrm{~nm}, \mathrm{D}_{\text {in }}=125 \mathrm{~nm}$ with a shell thickness of $10 \mathrm{~nm}$ for the Au nanoshells and Outer edge $=50 \mathrm{~nm}$, Inner edge $=42 \mathrm{~nm}$ with a wall thickness of $4 \mathrm{~nm}$ for the Au nanocages. These dimensions are similar to those materials used in the previous ${ }^{7}$ and current work. The DDA results are shown in Figure 5. Overall, Au nanoshells have a much greater extinction value than Au nanocages; however, the extinction of $\mathrm{Au}$ nanoshells is dominated by scattering. In contrast, the extinction spectrum of the $\mathrm{Au}$ nanocages is dominated by absorbance. As a result, at $800 \mathrm{~nm}$, Au nanocages have greater absorbance than $\mathrm{Au}$ nanoshells $\left(0.036\right.$ vs. $\left.0.027 \mu \mathrm{m}^{2}\right)$. Since PAT is an absorption-based technique, the greater absorption of the $\mathrm{Au}$ nanocages makes them more suitable than $\mathrm{Au}$ nanoshells for use as a contrast agent, as demonstrated in this study Another feature showed by this result is that Au nanocages have much narrower absorbance peak than Au nanoshells, and therefore more sensitive to laser wavelength.

\subsection{Results for dye-containing Nanoparticles}

The results for NIRex ${ }^{\mathrm{TM}}-4002$ are shown in Figure 6. Two PAT images of the cerebral cortex of a rat are presented in Figures $6 \mathrm{~A}$ and $6 \mathrm{~B}$. Figure $6 \mathrm{~A}$ shows the cerebral cortex imaging before the injection of nanoparticles while Figure 6B is the image after the injection of nanoparticles. Compared with Figure 6A in which only intrinsic optical contrast 
provides the image, Figure $6 \mathrm{~B}$, acquired after the administration of Au nanocages, shows the brain vasculature with greater clarity. With nanoparticles, the optical absorption of the blood was increased and the contrast between the vessels and the background brain tissues was enhanced. A differential image is shown in Figure 6C and was obtained by subtracting the amplitude of the pre-injection image (Figure 6A) from that of the post-injection image (Figure 6B). This image displays the distribution of differential optical absorption in the rat brain as induced by the exogenous contrast agent. The increases in optical absorption after nanopariticle injection were calculated. The signals were integrated over each image and then normalized to the signal integration of the reference image. The result is shown in Figure $6 \mathrm{C}$. The enhancement reaches about $50 \%$ after injection, and maintained for over 3 hour. The experiment was terminated because the unstable condition of the animal. Testing results for NIRex ${ }^{\mathrm{TM}}-4004$ is shown in Figure 7. These results are similar to that in Figure 6, but only the enhancement reaches about 25\% after injection, and reduced after that due to the clearance of nanopaticles from blood.
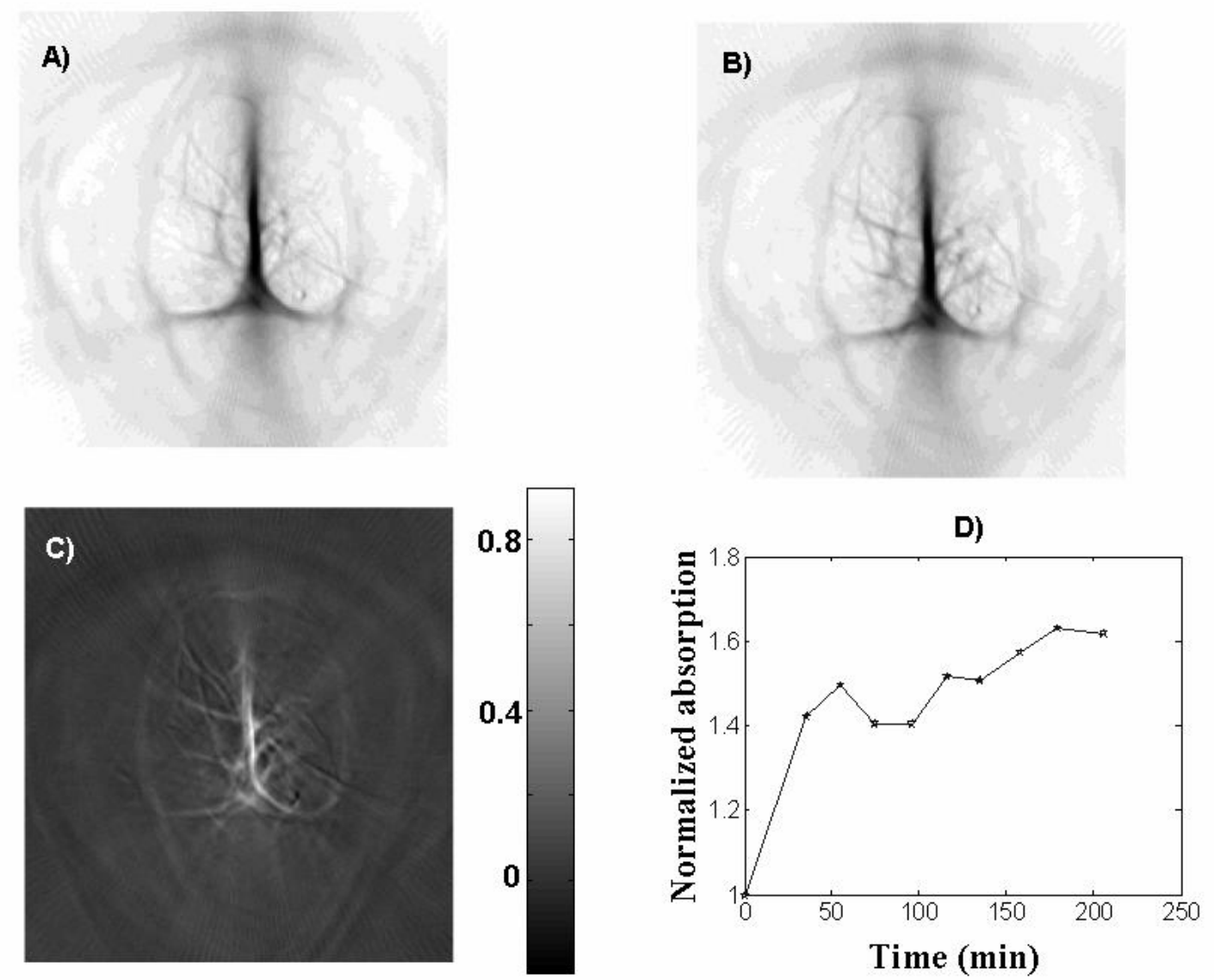

Figure 6 Sample NIRex ${ }^{\mathrm{TM}}-4002$ was injected. Non-invasive PAT imaging of a rat's cerebral cortex (A) before the injection of nanoparticles; (B) about 2 hours after the final injection of nanoparticles, which is the peak enhancement point. (C) A pixel-wise differential image (image B - image A) (D) The integrated absorption calculated from the experimental images. 

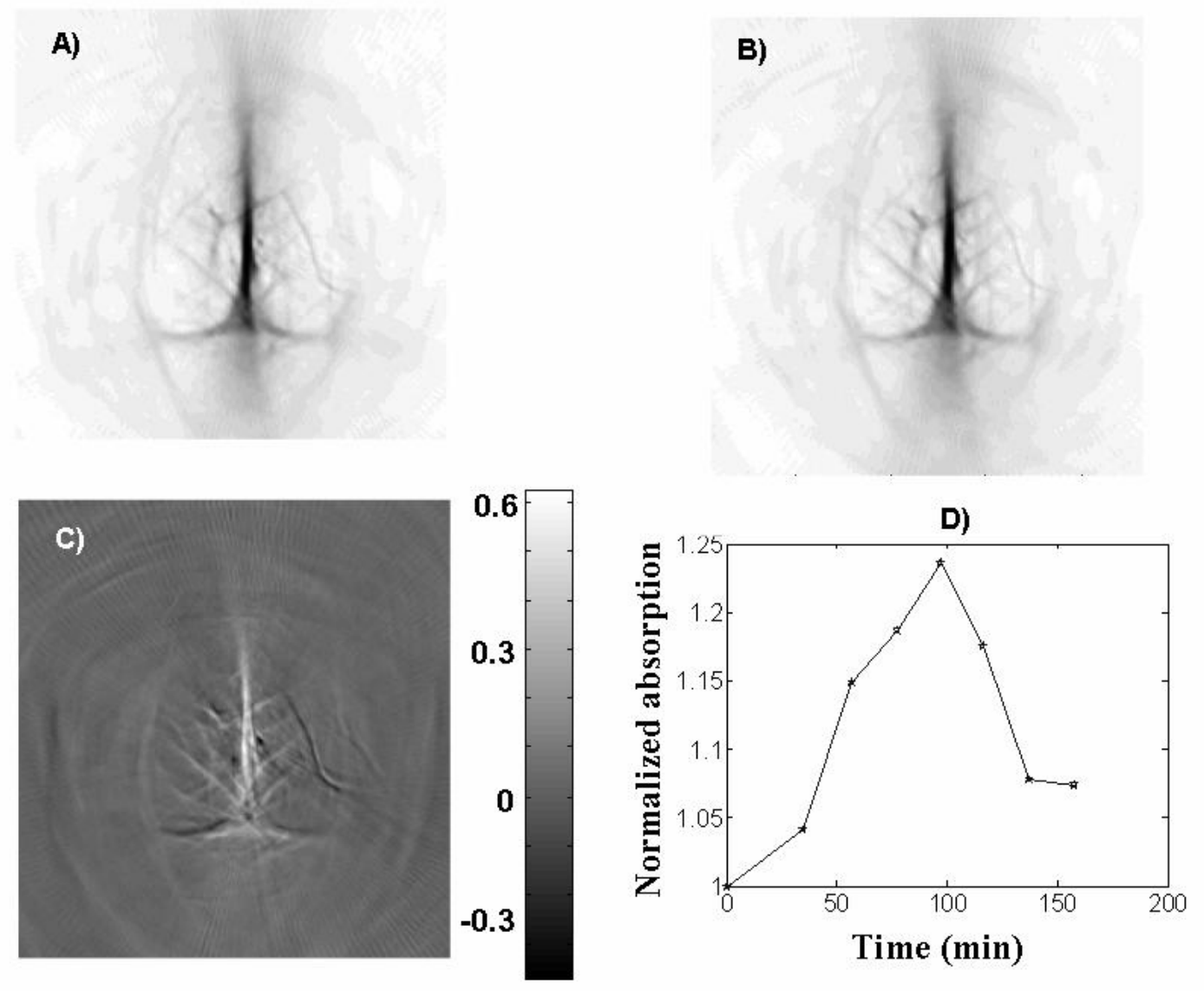

Figure 7 Sample NIRex ${ }^{\mathrm{TM}}-4004$ was injected. Non-invasive PAT imaging of a rat's cerebral cortex (A) before the injection of nanoparticles; (B) about 2 hours after the final injection of nanoparticles, which is the peak enhancement point. (C) A pixel-wise differential image (image B - image A) (D) The integrated absorption calculated from the experimental images.

\section{SUMMARY}

We have shown that Au nanocages and NIR dye-containing nanoparticles can be used in photoacoustic imaging to enhance the contrast between the blood vessels and the surrounding tissues. The enhanced contrast could allow more detailed vascular structures to be imaged at greater depths. Our results demonstrate that these nanoparticles are suitable contrast agents for imaging techniques based on optical absorption and in particular PAT. We envision that photoacoustic-based imaging using bioconjugated nanoparticles to target cancer cells could provide a new tool for tumor detection. 


\section{ACKNOWLEDGEMENTS}

This project was sponsored in part by National Institutes of Health Grant Nos. R01 CA106728 and R01 NS46214 (BRP) as well as a Director's Pioneer Award (5DP1OD000798 to Y. X.) and a grant from NSF (DMR-0451788 to Y. X.). Part of this work was performed at the UW Nanotech User Facility (NTUF), a member of the National Nanotechnology Infrastructure Network (NNIN) funded by NSF

\section{Reference:}

${ }^{1} \mathrm{Ku}, \mathrm{G}$. and Wang, L. V. "Deeply penetrating photoacoustic tomography in biological tissues enhanced with an optical contrast agent," Opt. Lett., 30, 507-509 (2005).

2 Wang, X., Pang, Y., Ku, G., Xie, X., Stoica, G. and Wang, L. V., "Non-invasive laser-induced photoacoustic tomography for structural and functional imaging of the brain in vivo," Nat. Biotechnol., 21, 803-806 (2003).

${ }^{3}$ Hoelen, C. G. A., de Mul, F. F. M., Pongers, R. and Dekker, A., "Three-dimensional photoacoustic imaging of blood vessels in tissue," Opt. Lett., 23, 648-650 (1998).

${ }^{4}$ Kruger, R. A., Reinecke, D. R. and Kruger, G. A., "Thermoacoustic computed tomography-technical considerations," Med.Phys., 26, 1832-1837 (1999).

${ }^{5}$ Oraevsky A. A. and Wang, L. V., Proc. SPIE, 6086, (2006).

${ }^{6}$ Xu, M. and Wang, L. V., "Photoacoustic imaging in biomedicine," Rev. Sci. Instrum., 77, 041101, 1-22. (2006).

${ }^{7}$ Wang, Y., Xie, X., Wang, X., Ku, G., Gill, K. L., O’Neal, D. P., Stoica, G. and Wang, L. V., Nano Lett., 4, 1689-1692 (2004).

${ }^{8}$ Wang, X., Ku, G., Wegiel, M. A., Bornhop, D. J., Stoica, G. and Wang, L. V., "Non-invasive photoacoustic angiography of animal brains in vivo with NIR light and an optical contrast agent," Opt. Lett. 29, 730-732. (2004).

${ }^{9}$ Licha, K., "Contrast agents for optical imaging," In Topics in Current Chemistry; Springer Berlin/Heidelberg, pp 1-29 (2002).

${ }^{10}$ Chen, J., Wiley, B., Li, Z. Y., Campbell, D., Saeki, F., Cang, H., Au, L., Lee, J., Li, X. and Xia, Y., “Gold nanocages: Engineering their structure for biomedical applications," Adv. Mater., 17, 2255-2261 (2005).

${ }^{11}$ Siekkinen, A. R., McLellan, J. M., Chen, J. Y. and Xia, Y., "Rapid synthesis of small silver nanocubes by mediating polyol reduction with a trace amount of sodium sulfide or sodium hydrosulfide," Chem. Phys. Lett., 432, 491-496 (2006).

${ }^{12}$ Chen, A. M. and Scott, M. D., "Current and future applications of immunological attenuation via pegylation of cells and tissue.," BioDrugs, 15, 833-847 (2001).

${ }^{13}$ Harris, L. M., Martin, N. E. and Modi, M. "Pegylation - A novel process for modifying pharmacokinetics," Clin. Pharmacokinet., 40, 539-551 (2001).

$14 \mathrm{Xu}, \mathrm{M}$., Xu, Y. and Wang, L. V., "Time-domain reconstruction algorithms and numerical simulations for thermoacoustic tomography in various geometries," IEEE Transactions on Biomedical Engineering, 50, 1086-1099 (2003). 